\title{
Distributed Coordination, Transportation \& Localisation in Industry 4.0
}

\author{
Ruben Van Parys ${ }^{1}$, Maarten Verbandt ${ }^{1}$, Marcus Kotzé ${ }^{2}$, Peter Coppens ${ }^{1}$, \\ Jan Swevers ${ }^{1}$, Herman Bruyninckx ${ }^{2}$, Johan Philips ${ }^{2}$, Goele Pipeleers ${ }^{1}$
}

\begin{abstract}
Factories of the future must be agile to adapt to rapidly changing customer needs, market volatility and shortened product life cycles. This requires flexibility in hardware and software at distinct levels of the factory and manufacturing process: multipurpose machines with fast change-overs, easy to use reconfigurable software and distributed decision-making are key. This paper leverages the new Industry 4.0 design principles to cope with these new manufacturing requirements: (i) distributed auction-based coordination allows local decision-making and task allocation, (ii) distributed model predictive controlbased transportation enables free space collision avoidance of automated guided vehicles (AGVs), and (iii) distributed visionbased localisation provides scalable and dynamic position information of key resources on the factory floor. Furthermore, these contributions are brought together in a lab-scale reconfigurable manufacturing system to showcase modularity and distributed decision-making at several levels of a manufacturing process' logistics.
\end{abstract}

\section{INTRODUCTION}

Novel manufacturing paradigms have been introduced to cope with rapidly changing customer needs, market volatility and shortened product life cycles, which factories of the future are faced with. Depending on application domain and scope, these developments are addressed as Reconfigurable Manufacturing Systems (RMS) [1], Industry 4.0 [2] or Smart Factories [3]. All aim at increasing modularity, scalability and reconfigurability of manufacturing systems. For clarity, in this paper, Industry 4.0 is used to denote solutions to this evolution.

Although the new manufacturing trends have been extensively presented, many publications are restricted to a highlevel description providing general design principles [1]. Other work mainly focuses on the logistics and general coordination of manufacturing processes [4], [5]. Low-level components

This work benefits from KU Leuven-BOF PFV/10/002 Centre of Excellence: Optimization in Engineering (OPTEC); from the projects G0C4515N and G.0915.14 of the Research Foundation-Flanders (FWO-Flanders); from Flanders Make SBO ROCSIS: Robust and Optimal Control of Systems of Interacting Subsystems; from KU Leuven IOF Kennisplatform Transition; from European Union's Horizon 2020 project RobMoSys (H2020-732410), Composable Models and Software for Robotics Systems-of-Systems, 7th Framework Programme project PicknPack (FP7-311987); and from the KU Leuven Research project C14/15/067: B-spline based certificates of positivity with applications in engineering. Ruben Van Parys is a PhD fellow of FWOFlanders. Flanders Make is the Flemish strategic research centre for the manufacturing industry.

${ }^{1}$ MECO Research Team, Department Mechanical Engineering, KU Leuven and the DMMS core lab, Flanders Make, Leuven, Belgium (email: ruben.vanparys@kuleuven.be).

${ }^{2}$ Robotics Research Team, Department Mechanical Engineering, KU Leuven. constituting these processes, commonly addressed as cyberphysical systems (CPS), are often assumed to meet certain requirements without explaining how this is achieved in practice [6].

This paper focuses on three main components within a manufacturing process: (i) coordination, (ii) transportation and (iii) localisation. For each component a modular architecture with distributed decision-making is proposed and implemented to meet Industry 4.0 design principles on scalability, reconfigurability and robustness. Methodological innovations were realized to enable distributed decision-making on the coordination and transportation level. Furthermore, technological contributions were made on the implementation of a modular localisation system with a semi-automated calibration procedure, the development of hardware and software for each component, and the integration of all components into a fully operational demonstration.

The following subsections describe the state-of-the-art for each of the components and highlight the innovative contributions of this paper.

\section{A. Coordination}

Traditionally, manufacturing environments are coordinated in a centralised way. Although such a framework renders the manufacturing system convenient when it comes to supervising different tasks, it ranks low in terms of flexibility [7] compared to distributed or hybrid architectures, which offer a higher degree of reconfigurability. Holonic systems form a subset of these distributed architectures and are based on the concept of autonomous cooperating agents, called holons [8]. The holonic approach to the coordination distributes coordination and decision-making among holons allowing them to solve local problems locally. Scaling the system is therefore more straightforward compared to traditional centralised coordination.

Holonic coordination knows two common implementations, Product-Resource-Order-Staff Architecture (PROSA) [9] and ADAptive holonic COntrol aRchitecture (ADACOR) [10]. Some related work argues that ADACOR is a partial instantiation of PROSA, with reduced scope but increased functionality [11].

In this work, ADACOR is chosen as a baseline. In an Industry 4.0 setting, the coordination has to deal with decisionmaking which is distributed over several modules. To overcome this difficulty, ADACOR is extended with an auctionbased deliberation, using contract nets, to explicitly allocate 
appropriate resources for each task. Moreover, in the presented setup, the work of [12], where ADACOR was applied to conveyor belt systems, is extended to free space transportation.

\section{B. Transportation}

Efficient and safe product transportation is a key element in a successful manufacturing system. Transportation trends have gradually shifted from rigid conveyor belts towards automated guided vehicles (AGVs). Although AGVs enable flexible transportation, they are often constrained to follow dedicated tracks, separated from humans [13]. Free space motion planning unlocks their true potential, as it enables AGVs to navigate while obeying system limitations and avoiding nearby collisions.

When multiple AGVs are present, a distributed control architecture is preferable. Each AGV computes its own motion trajectory and communicates with its peers to avoid collisions. Increased modularity, scalability and robustness are clear advantages of this approach compared to a central motion coordination scheme. Distributed motion control is a well-investigated topic in robotics. Many state-of-the-art approaches use the concept of velocity obstacles to achieve mutual collision avoidance [14], [15] but fail to optimise a free space motion trajectory within a control horizon. In contrast, a distributed model predictive control (DMPC) approach enables optimal free trajectory computations within the control horizon taking into account a vehicle's limitations and collision avoidance [16], [17].

To meet all application requirements, free space transportation using DMPC is chosen to control the AGVs. In order to robustify and facilitate the mutual collision avoidance of AGVs, an earlier presented DMPC approach [18]-[20] is extended by means of priority rules. In addition to methodological contributions, hardware and software for a lab-scale AGV is developed [21] and presented in this paper.

\section{Localisation}

Free space motion planning has a strong impact on localisation, as it now has to cover the entire workspace rather than predefined tracks. Various approaches to tackle this problem have been proposed. Currently LIDAR-based systems have found wide acceptance in industry. Cheap reflectors spread across the factory provide landmarks from which the LIDAR's position is determined [22]. This facilitates positioning, but requires an expensive sensor for each object of interest. Because wireless technology has become affordable, a new class of indoor localisation systems has emerged [23]-[25]. However, only ultra-wide band technology is currently able to provide the accuracy needed for navigation as it suffers less from the inevitable reflections indoor [24]. Moreover, the amount of required sensors once again depends on the number of objects, possibly rendering the overall system expensive.

More widespread throughout academia are vision-based systems. Their high information density makes them suited for a variety of tasks such as obstacle detection, product identification or object tracking, which implies a high reconfigurability. With fixed cameras, the required number of sensors is decoupled from the number of agents, creating a scalable solution. These two properties make a top-view vision-based localisation system ideal in an Industry 4.0 framework. Examples of such systems are widely available within the robotics literature, especially in a robotic soccer context [26]-[28]. These provide basic insight on how to achieve accurate localisation and identify several agents within the image. Another noteworthy vision-based localisation system is discussed in [29]. Here, a network of cameras is installed to observe the scene from different viewpoints, which allows elimination of perspective effects.

In order to obtain a distributed and scalable indoor localisation system, this work proposes the use of a series of ceiling camera modules monitoring the work floor. Extra modules are readily added to comply with a changing work floor thanks to a semi-autonomous calibration procedure. This procedure, together with the localisation algorithms, is part of ProjectEagle, a software package [30] developed within the context of this work.

\section{Problem FORMULATION}

To assimilate the Industry 4.0 design principles, this paper aims at developing a fully modular manufacturing system where online distributed decision-making is integrated on all levels. Such system allows to be reconfigured online, e.g. to handle different product families, and is easily scaled to meet capacity changes by adding or removing modules. In terms of robustness, a failure of one module does not result in a failure of the complete manufacturing system as other modules can take over tasks, and a failed module is easily replaced.

The considered setting is depicted by Figure 1 and presents a modern manufacturing system with different interacting intelligent modules. Automated guided vehicles (AGVs) collect and deliver products at mobile work stations, e.g. an assembly cell, a CNC machine or a bin-picker unit. A modular localisation system is installed to detect AGVs and work stations and broadcasts their position information, allowing AGVs to localise themselves, to plan motion trajectories, and to dock near work stations. Finally, a holonic coordination framework gathers tasks, queries for available resources and dispatches tasks to appropriate work stations and AGVs. Although the proposed methodologies are by design applicable to larger, more complex setups, the limited number of available resources led to a lab-scale demonstration as depicted in Figure 1 and presented in Section VI.

The following three sections detail on the implementation of distributed decision-making on three levels of the considered manufacturing process: (i) distributed auction-based coordination allows local decision-making and task allocation, (ii) distributed model predictive control-based transportation enables free space collision avoidance of AGVs, and (iii) distributed vision-based localisation provides scalable and dynamic position information of key resources on the factory floor. 


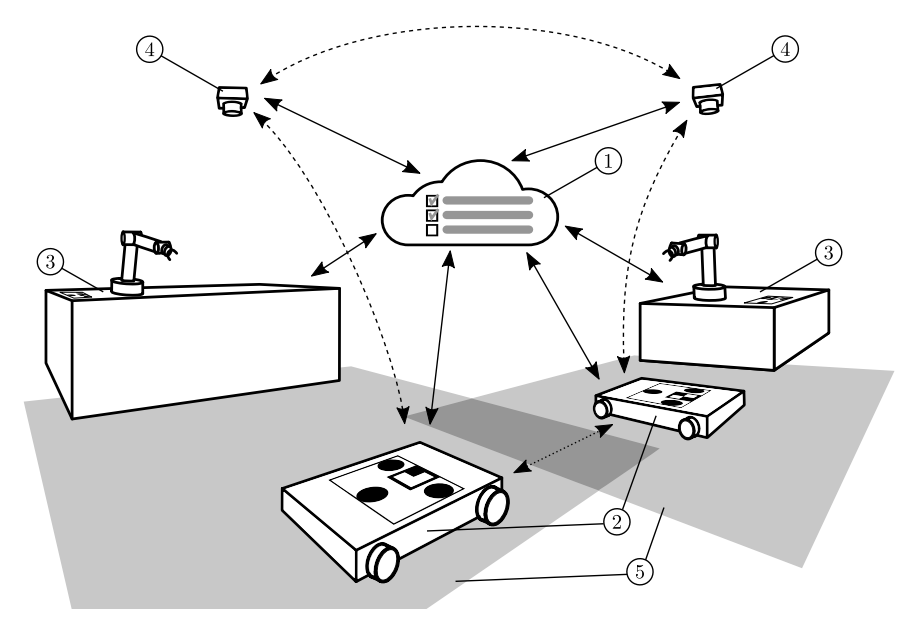

Fig. 1: Overview of the considered setting with (1) a holonic coordination system, (2) automated guided vehicles, (3) work stations and (4) a localisation system composed of different camera modules with their corresponding field-of-view depicted by (5). Arrows indicate the communication flow between the different modules.

\section{DistRIBUTED AUCTION-BASED COORDINATION}

The coordination module is responsible for governing various activities within the manufacturing system. Here, an ADACOR-based [10] holonic coordination strategy which allows dynamic reconfiguration is proposed. The implemented software is an extension of the coordination of a reconfigurable conveyor belt system [12] to an entire work cell with free space navigation.

\section{A. Holonic design principle}

In manufacturing systems, a holon is an autonomous and cooperative entity, able to transform, transport, store or validate information of physical objects. One of the key notions in holonic coordination is to have a close relationship between the coordination software architecture and the system hardware. Therefore, each physical module in the manufacturing system is associated with a corresponding holon (in software and hardware). Within this distributed architecture, modularity on the physical level is reflected also on the software level. Furthermore, holons have the ability to negotiate with each other to achieve individual and common objectives. This way holonic systems autonomously find feasible (if not optimal) approaches to complete production steps, even after reconfiguration of the manufacturing system.

This work employs three classes of holons: the supervisor holon, operational holon and task holon. The supervisor holon is responsible for launching and terminating holons, for interholon coordination and for global optimisation. An operational holon is responsible for managing a physical module in the manufacturing system. In this work two module types are considered, an AGV and a work station. Therefore two types of operational holons are introduced, an AGV holon and work station holon. Each physical module has its own corresponding operational holon deployed in the coordination software. A task holon is responsible for coordinating a specific task in the manufacturing system. It does this by negotiating with the operational holons to perform the required subtasks.

\section{B. Task allocation}

The coordination module is mainly responsible for processing incoming tasks by allocating appropriate resources. Whenever an operator submits an order, the supervisor holon creates a corresponding task holon, which starts solving the allocation problem by

1) querying stock of each work station;

2) calculating a minimal combination of work stations for this task;

3) allocating the required work stations, or terminating if no solution is found.

Currently, the selection process only takes into account current stock count. For future work, also current or predicted workload of each work station should be included.

After selecting the work stations, the task holon launches an auction to determine which $\mathrm{AGV}$ will be in charge of transportation by

1) issuing a request for proposals to all AGV holons;

2) selecting the most competitive bid, which is based on an estimated task execution time;

3) allocating the AGV that won the auction.

To determine the estimated task execution time, AGV holons solve a Travelling Salesman Problem to acquire the shortest route, estimate time to complete that route, add expected time spent at each work station and finally add estimated remaining time of the current task list. An improvement would be that AGV holons factor in the required work station's current operations and uses this information in calculating a route plan and time estimation, similar to what the task holon should do when selecting work stations.

The task execution phase is completed by the following sequence:

1) AGV holon issues the waypoint location to the corresponding AGV;

2) on arrival at a work station, the AGV holon notifies the work station holon;

3) work station performs its specific subtask;

4) work station holon signals the AGV holon such that it can command the AGV to move to the next waypoint;

5) if all waypoints are exhausted, the AGV returns to its home position where it does not interfere with other AGVs.

The holonic design principle facilitates the combination of discrete (topological) planning and global coordination with continuous (motion) planning and local coordination of each AGV. This hybrid approach allows local decision-making and problem solving, making the overall solution more scalable.

\section{DISTRIBUTED MODEL PREDICTIVE CONTROL-BASED TRANSPORTATION}

Transportation of products is accomplished by a fleet of small in-house developed AGVs. To acquire a modular and 


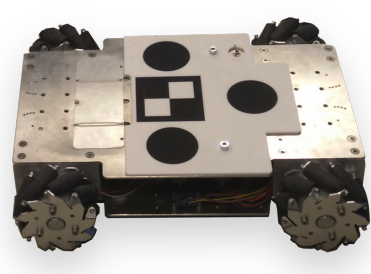

(a)

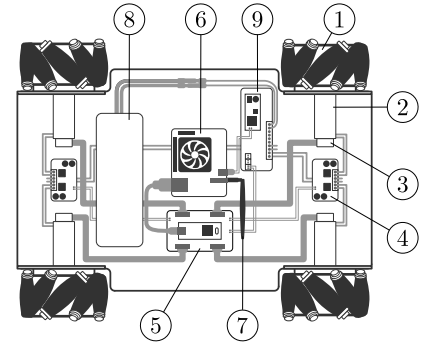

(b)
Fig. 2: In-house developed automated guided vehicle. A picture of the setup is illustrated in (a), while (b) gives an overview of its components: (1) a Mecanum wheel, (2) a DC motor, (3) an encoder, (4) a dual DC motor drive, (5) the Teensy microcontroller, (6) the Odroid XU4, (7) the WiFi dongle, (8) a 24 V LiPo battery and (9) a voltage regulator board.

scalable system, the distributed motion planning approach of [18], [19] is extended with priority rules: each AGV contains a computing device that generates its own motion trajectory and communicates with its peers to avoid collisions without relying on the high-level coordination module. This section first gives an overview of the developed AGV, afterwards the motion planning approach is explained in detail.

\section{A. Hardware overview}

Figure 2 illustrates the developed vehicle. An AGV is equipped with four independently driven Mecanum wheels, which renders the system holonomic [31]. Four DC motors with encoders drive the wheels and their velocities are controlled using a Teensy micro controller. This device is connected via USB to an Odroid XU4 single-board computer. This small computer is provided with a WiFi dongle, which allows it to communicate with other modules in the manufacturing system.

The Odroid XU4 has sufficient computing power to perform different control-related tasks simultaneously. First of all it implements a Kalman filter that merges absolute pose information received from the camera modules with its local encoder measurements to retrieve an accurate state estimate at a rate of $100 \mathrm{~Hz}$. Second, the motion planning algorithm described in Section IV-B is executed periodically by the Odroid with a receding horizon at a rate of $2 \mathrm{~Hz}$. The generated motion trajectories are tracked by a low-level feedback controller running at $100 \mathrm{~Hz}$ that generates velocity setpoints for the Teensy micro controller. All software is implemented within the real-time Orocos Toolchain framework [32] and is freely available online [21].

\section{B. Free space motion planning}

The motion planning of an AGV is performed using an optimisation-based approach to incorporate system limitations and collision-avoidance constraints in a systematic way. The approach is based on [18], [19] and provides every AGV with a distinct controller that computes its own trajectory. AGVs communicate their planned trajectories to mutually avoid collisions. Trajectories are generated in an MPC fashion. Hence, at every control period, an AGV's optimal control problem is solved over an updated control horizon, starting from an estimate of its current state. In this way, an AGV can account for possible disturbances and changes in the environment, due to e.g. other moving AGVs.

1) Optimal control problem: Every control period, an AGV solves an optimal control problem that searches for its position trajectory $q(\cdot):[0, T] \rightarrow \mathbb{R}^{2}$ expressed in the ground plane, to steer it from a current position $q_{0}$, at $t=t_{0}$, towards a target destination $q^{*}$. Optimal trajectories are obtained by minimising an objective of the form

$$
\int_{t_{0}}^{t_{0}+T}\left\|q(t)-q^{*}\right\|_{1} d t
$$

which will steer the AGV as close to the destination $q^{*}$ as possible during the control horizon $T$. As the $L^{1}$-norm puts relatively large weight on small residuals, objective (1) results in a motion with low overshoot [33]. The AGV is considered to be a velocity-steered holonomic vehicle such that its input is $u=\dot{q}$. To incorporate actuator limitations and to generate smooth trajectories, bounds on $\dot{q}$ and $\ddot{q}$ are imposed. Additionally, collision-avoidance constraints with the environment and other AGVs are introduced which are further explained in Section IV-B2. With $f$ representing the collisionavoidance constraints, the optimal control problem is written as:

$$
\begin{array}{cl}
\underset{q(\cdot)}{\operatorname{minimize}} & \int_{0}^{T}\left\|q(t)-q^{*}\right\|_{1} d t \\
\text { subject to } & q\left(t_{0}\right)=q_{0}, \quad \dot{q}\left(t_{0}\right)=\dot{q}_{0}, \\
& u_{\min } \leq \dot{q}(t) \leq u_{\max }, \\
& \dot{u}_{\min } \leq \ddot{q}(t) \leq \dot{u}_{\max } \\
& f(q(t)) \leq 0, \quad \forall t \in[0, T] .
\end{array}
$$

An AGV's orientation is controlled independently of its position as the vehicle is holonomic, system limitations are imposed on coordinate level and the AGV is approximated by a circle for collision avoidance. The orientation is steered towards its desired state using a trapezium velocity profile.

2) Collision avoidance: Similar as in [18]-[20], obstacle avoidance constraints are constructed by imposing the existence of a separating hyperplane between the AGV and the obstacle. Note that this construction can only separate convex shapes [33]. Suppose the geometry of an AGV is approximated by a circle with centre $q$ and radius $r$, while an obstacle $j$ is represented by a circle with centre $q_{j}$ and radius $r_{j}$. Enforcing the separation of both shapes by a line $\left\{p \in \mathbb{R}^{2} \mid a_{j}^{T} p=b_{j}\right\}$ is achieved with the following set of constraints:

$$
\begin{aligned}
a_{j}(t)^{T} q(t)-b_{j}(t) & \geq r, \\
a_{j}(t)^{T} q_{j}(t)-b_{j}(t) & \leq-r_{j}, \\
a_{j}(t)^{T} a_{j}(t) & \leq 1, \\
\forall t & \in[0, T] .
\end{aligned}
$$

To avoid collisions at all times, the separating line is allowed to change over time and both $a_{j}(\cdot)$ and $b_{j}(\cdot)$ are introduced as time-dependent optimisation variables. In case of a dynamic 
environment, predictions concerning the obstacle's motion are incorporated using a time-dependent trajectory $q_{j}(\cdot)$.

Apart from obstacles, an AGV should also avoid other AGVs in the environment. This is enforced by allowing AGVs to communicate their planned trajectories and using similar constraints as (3) where $q_{j}(\cdot)$ is replaced by the trajectory of the peer to be avoided. It is however not recommended that two AGVs try to avoid each other simultaneously. This generally leads to oscillatory behaviour or livelocks. Instead, AGVs avoid each other based on a priority rule. A lower priority vehicle uses communicated trajectories from higher priority vehicles to avoid them properly. Priority levels of AGVs change event-based. A vehicle that started moving earlier gets a higher priority than a vehicle that started later. On the other hand, AGVs that are docked near a work station or that are at their home position, get the highest priority as it is undesirable that they change position to avoid other AGVs. An AGV can track down its peers' priority levels and can determine its own as each AGV is broadcasting its priority changes on the network. Prioritising a vehicle with respect to others can however lead to global suboptimal solutions. Systematic approaches to reduce this suboptimality are presented in [20].

3) Spline parametrisation: Optimal control problem (2) is infinite dimensional, comprising both infinitely many optimisation variables and constraints, as the optimisation variables are functions and they must satisfy constraints at all time instants. To cope with the infinitely many optimisation variables, the time-dependent variables are approximated by piecewise polynomials and are parametrised in a B-spline basis [34]:

$$
\hat{q}(t)=\sum_{i=1}^{m} \mathrm{q}_{i} b_{i}(t),
$$

with B-spline basis $b=\left[b_{1}, \ldots, b_{m}\right]^{T}$ and B-spline coefficients $q=\left[q_{1}, \ldots, q_{m}\right]$. The main reason for adopting the $\mathrm{B}$-spline basis is the so-called convex hull property: as the $\mathrm{B}$-splines are positive and sum up to 1 , a spline is always contained in the convex hull of its B-spline coefficients. This way, bounds on a spline function can be enforced by imposing them on the coefficients:

$$
\mathbf{q} \leq 0 \Rightarrow \hat{q}(t) \leq 0, \forall t \in[0, T] .
$$

Because (anti-)derivatives and polynomial functions of splines are splines as well, also polynomial constraints on spline trajectories and their (anti-)derivatives can be handled in the same way. For holonomic vehicles all constraints are naturally polynomial in the motion trajectories $q$ and their derivatives. For details on how to account for nonholonomic vehicles, the reader is referred to [18], [19]. A spline parametrisation is employed for $q(\cdot), a_{j}(\cdot)$ and $b_{j}(\cdot)$ and problem (2) is reformulated in terms of the corresponding spline coefficients.

The spline-based motion planning approach is implemented as part of a software toolbox named Optimal Motion Generation-tools [35]. The toolbox is written in Python and uses CasADi [36] as symbolic framework and interface to solvers. The goal of OMG-tools is to facilitate the modelling,

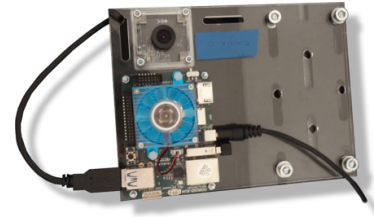

(a)

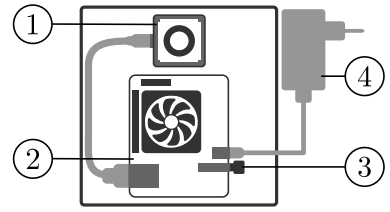

(b)
Fig. 3: Camera module. A picture of the module is illustrated in (a), while (b) gives an overview of its components: (1) the Odroid oCam 5MP, (2) the Odroid XU4, (3) a WiFi dongle and (4) an AC adapter.

simulation and deployment of spline-based motion problems. The toolbox is able to export a library which is imported in the software framework running on an AGV's Odroid XU4.

\section{DISTRIBUTED VISION-BASED LOCALISATION}

This section focuses on the development of a distributed vision-based localisation system, ProjectEagle [30]. The system consists of a series of camera modules equipped with a single-board computer. To provide consistent measurements, each module is put through a calibration procedure. To streamline this process, a Graphical User Interface (GUI) has been designed, which is freely available online [30]. The GUI allows the user to easily add new camera modules to the network. Next, the user starts the calibration procedure after which the parameters are stored on the module. The latter allows all camera modules to locally run a localisation algorithm, without the need to interact with one another. This has several advantages. First, the available computational capacity is used to the maximum, which is characteristic for distributed systems. Second, every module is capable of providing globally consistent measurements by itself. This implies scalability of the system: modules are easily added to or removed from the system to adapt the visible area.

The following subsections give a more detailed explanation of the selected hardware, the calibration procedure and the localisation of a distinct marker within the video stream.

\section{A. Hardware overview}

Monitoring the workspace is realised by a set of camera modules, attached to the ceiling. The module is illustrated in Figure 3 and consists of three main parts. An Odroid oCam $5 \mathrm{MP}$, equipped with a wide angle lens, provides a video stream of the workspace. This video stream is transmitted via USB to an Odroid XU4 single-board computer, capable of extracting position information at a rate of $10 \mathrm{~Hz}$. By means of a $\mathrm{WiFi}$ dongle, the Odroid XU4 is able to transmit the measurements to other modules in the network.

\section{B. Calibration}

As with all camera-based localisation systems, a good calibration procedure is vital to the accuracy of the position measurements. The camera calibration is usually split in two parts: the intrinsic and extrinsic calibration. 
The intrinsic calibration [37] is concerned with finding the parameter values of the selected camera model, in this case the standard pinhole camera model:

$$
\lambda_{p} p=K_{c} p,
$$

with ${ }_{p} p=[u, v, 1]^{T}$. Given a scaling factor $\lambda$, this model enables the mapping of pixel coordinates $[u, v]^{T}$ to coordinates ${ }_{c} p \in \mathbb{R}^{3}$, expressed in a frame $\{c\}$ attached to the camera. The pinhole model is characterised by the camera matrix $K$, which is determined from a set of training data, usually a series of chessboard pictures. To reduce the effect of lens distortions, $[u, v]^{T}$ is obtained by first applying a nonlinear transformation to the distorted pixel coordinates. The nonlinear distortion model is determined together with $K$.

The extrinsic calibration searches for the transformation that converts camera coordinates to world coordinates. Since the location of the world frame is arbitrary, it is fixed through the calibration of the first camera module. Figure 4 illustrates how the world frame $\{w\}$ is chosen. Its origin $o^{\prime}$ lies on the intersection of the ground plane $\gamma$ and the $z$-axis of $\{c\}$. The $x^{\prime}$-axis of $\{w\}$ follows the intersection of $\gamma$ with the plane spanned by the $x$ - and $z$-axis of $\{c\}$. The $z^{\prime}$-axis is chosen to be perpendicular to $\gamma$, pointing upwards and the $y^{\prime}$-axis is determined such that $\{w\}$ is a right-handed frame. Given the equation of the ground plane

$$
\gamma=\left\{{ }_{c} p \in \mathbb{R}^{3} \mid g^{T}{ }_{c} p=h\right\},
$$

where $g=\left[g_{x}, g_{y}, g_{z}\right]^{T}$, the origin ${ }_{c} O$ and unit vectors ${ }_{c} e_{x},{ }_{c} e_{z}$ are mapped to ${ }_{c} o^{\prime},{ }_{c} e_{x^{\prime}},{ }_{c} e_{z^{\prime}}$ respectively and their coordinates expressed in $\{c\}$ are

$$
\begin{aligned}
{ }_{c} o^{\prime} & =\left[0,0, \frac{h}{g_{z}}\right]^{T}, \\
{ }_{c} e_{x^{\prime}} & =\left[\frac{\left|g_{z}\right|}{\sqrt{g_{x}^{2}+g_{z}^{2}}}, 0,-\frac{g_{x}}{\sqrt{g_{x}^{2}+g_{z}^{2}}} \operatorname{sign}\left(g_{z}\right)+\frac{h}{g_{z}}\right]^{T}, \\
{ }_{c} e_{z^{\prime}} & ={ }_{c} o^{\prime}-\frac{g}{\|g\|_{2}} \operatorname{sign}\left(g_{z}\right) .
\end{aligned}
$$

Using the mapping of these three points, the transformation matrix ${ }_{w}^{c} T$ from $\{w\}$ to $\{c\}$ is easily determined [38].

For every subsequent camera, a slightly different approach is used to perform the extrinsic calibration since every new camera added to the system should be calibrated with respect to the already established world frame $\{w\}$. To this end, a small overlap between the fields of view of the new camera and one camera which has already been extrinsically calibrated, is introduced. A series of chessboard pictures taken from the two cameras now provides two point clouds: one in the world coordinate frame and one in the coordinate frame of the new camera. These point clouds are identical up to a rigid transformation, which is exactly the transformation from the world to the new camera. Once more, the technique of [38] is employed to compute this transformation.

Important to note is that every camera stores its own transformation from the world to its own coordinate system.

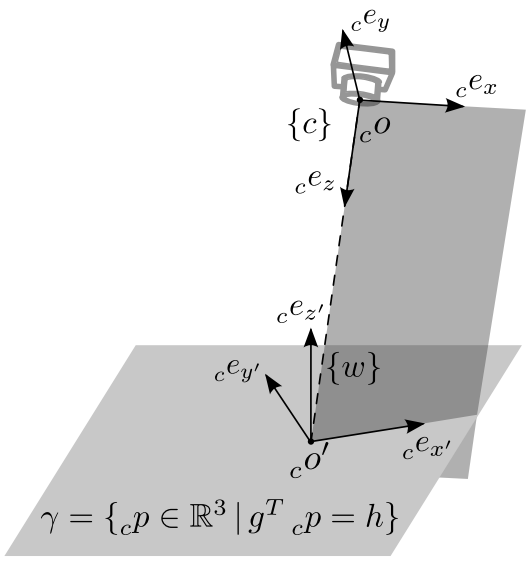

Fig. 4: Choice of the world coordinate frame based on the coordinate frame of the first camera.

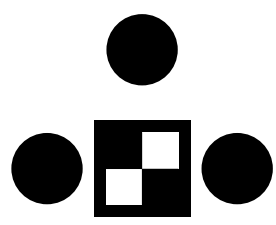

Fig. 5: Example marker for the localisation system.

By doing so, every camera module can function independently while providing consistent measurements.

\section{Localisation}

To obtain a reliable localisation at a sufficient rate, a distinct marker is installed on each module for which position information is desired. The marker, depicted in Figure 5 , consists of three disks in a isosceles triangle configuration, since the overall positioning accuracy of a series of small disks is found to outperform one bigger disk [39]. Along its centreline, a grid of black and white patches is added. This grid forms a 4-bit code to uniquely identify each marker. Using only black and white colours renders the system more robust to illumination changes without intricate correction schemes as proposed in [40].

A software implementation to localise this type of marker is available at [30]. The algorithm starts with a background subtraction procedure followed by thresholding the resulting image. An erosion-dilation operation takes care of noise suppression [41]. This yields a series of regions corresponding to unidentified objects traversing the workspace, making the search for markers more efficient. A blob detection algorithm is used to detect the distinct black disks of the marker. All possible combinations of blobs are tested against the properties of the isosceles triangle, rejecting false-positive blobs and returning the marker's position. Since the disks are not arranged in an equilateral triangle configuration, it is possible to determine which blobs correspond to the left, right and top disks. This allows blindly sampling the identification patches to determine the marker's ID.

To transform the blob's pixel coordinates to world coordinates, depth information is required. It is assumed that 
each marker moves in a specific plane, $\left\{{ }_{w} p \in \mathbb{R}^{3} \mid k^{T}{ }_{w} p=l\right\}$, which is typically parallel to the ground plane. Given the pixel coordinates ${ }_{p} p_{b}=\left[u_{b}, v_{b}, 1\right]^{T}$ of a blob, its corresponding coordinates ${ }_{w} p_{b}$ in $\{w\}$ are determined by solving the system of equations (4) for $\lambda$ and ${ }_{w} p_{b}$.

$$
\begin{aligned}
\lambda_{p} p_{b} & =K{ }_{w}^{c} T_{w} p_{b} \\
k^{T}{ }_{w} p_{b} & =l
\end{aligned}
$$

From the global coordinates of the three blobs, a module's position and orientation is derived.

\section{EXPERIMENTAL VALIDATION}

The strategies in coordination, transportation and localisation presented in the previous sections are combined in a small-scale warehousing application described and demonstrated below.

\section{A. Application overview}

To demonstrate the capabilities of the presented modular manufacturing system, a small-scale warehousing application is presented. A sketch of the developed setup is shown in Figure 1. Orders consist of a series of products to be delivered at a specified drop-off point, and are provided by an operator via a Graphical User Interface (GUI). Two AGVs, equipped with a basket in the back and front, take care of the transportation of products. They pick up products at the two work stations. Each work station contains a stock of different products and a UR5 robotic manipulator that is able to pick up products and drop them into an AGV's basket. Two camera modules are attached to the ceiling to span a visible area of $4.5 \mathrm{~m} \times 5.5 \mathrm{~m}$.

The motion of the UR5 robotic manipulator is taught a priori by demonstration which allows to easily reconfigure a work station's task. Since intelligent product picking lies outside the scope of this work, small wooden boxes are used to represent virtually different products. Similar to the AGVs, each work station carries a marker. This allows the localisation system to track the stations and broadcast their position on the network. This makes on-the-fly reconfiguration of the work floor possible without any form of intervention. Each AGV has all information to successfully avoid collisions with the stations or dock near them.

Communication is accomplished using the network library Zyre [42] and deployed on a local wireless network. It offers automatic peer discovery, peer-to-peer communication and message brokerage.

\section{B. Demonstration}

Experiments were carried out on the developed system and are demonstrated in a self-explanatory vided 1 Figure 6 highlights a few interesting situations which arise during the demonstration. The first order consists of two products. Since none of the work stations has both products, the responsible AGV has to stop at both work stations. Based on the received bids for the task, the coordination module selects one of the two AGVs which are idling on their home positions (Figure 6a. While the selected AGV, labelled AGV 1, is busy
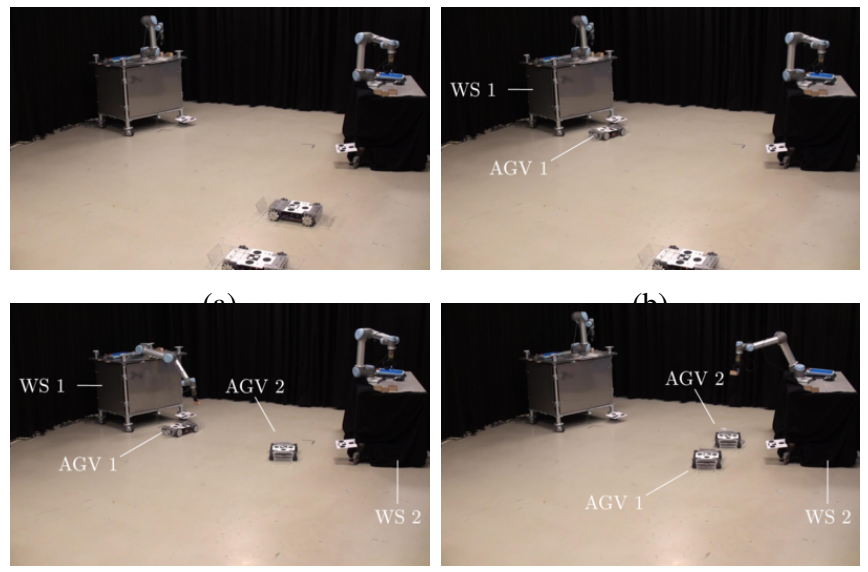

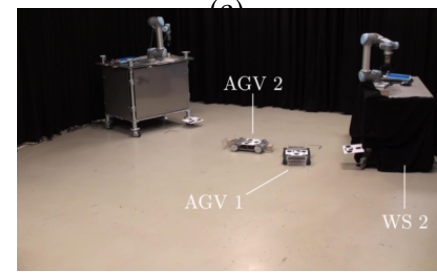

(e)

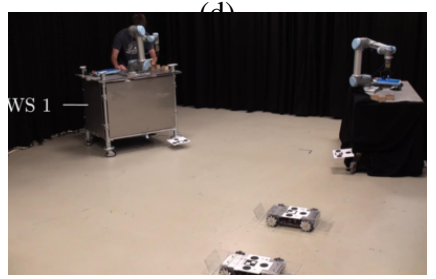

(f)
Fig. 6: Series of interesting situations which arise throughout the demonstration.

collecting the first order at work station WS 1 (Figure 6b), a second order has been dispatched, which is logically granted to AGV 2. This order requires products which are available at work station WS 2 (Figure 6c). While AGV 2 is being loaded at WS 2, AGV 1 has finished its task at WS 1 and approaches WS 2 (Figure 6d). Thanks to the priority-based collision avoidance, natural queuing arises: AGV 2, which is being loaded, has the highest priority and is avoided by AGV 1. As AGV 2 is located at the target position of AGV 1, the latter can only wait until AGV 2 leaves its current position. This happens when AGV 2 is finished at WS 2. As AGV 1 was already moving when AGV 2 was commissioned to leave its docking position, AGV 1 gets priority over AGV 2 and is avoided by the latter while it approaches the docking point of WS 2 (Figure 6e). Once both AGVs have accomplished the orders, work station WS 1 is relocated (Figure 6f) and the experiment is repeated to demonstrate the reconfigurability and flexibility of the developed setup.

\section{CONCLUSION}

This paper presented a lab-scale reconfigurable manufacturing system to showcase an integrated solution for factories of the future, leveraging Industry 4.0 design principles. Effort has been spent to introduce modularity and distributed decisionmaking on different system levels to retrieve a highly reconfigurable setup: (i) On localisation level, reconfigurability is obtained by combining a modular vision system with a swift calibration procedure which allows to easily scale the visible area by adding or removing camera modules. Furthermore, the continuous broadcasting of environmental information allows

\footnotetext{
${ }^{1}$ https: //youtu.be/txkjt $004 \mathrm{mwU}$
} 
other modules to adapt their behaviour online on possible changes in the system; (ii) On transportation level, free space motion planning and distributed model predictive control allow AGVs to easily adapt their trajectories when required. By using a distributed control approach, AGVs can locally solve conflicts by mutually coordinated collision avoidance. Finally, (iii) a distributed holonic coordination approach projects physical modularity to the software architecture which allows to incorporate reconfiguration of modules directly on coordination level.

A demonstration of the fully integrated manufacturing setup shows its capabilities and reconfigurability. Moreover, the software of the different submodules is available online.

\section{REFERENCES}

[1] Y. Koren, U. Heisel, F. Jovane, T. Moriwaki, G. Pritschow, G. Ulsoy, and H. Van Brussel, "Reconfigurable manufacturing systems," CIRP AnnalsManufacturing Technology, vol. 48, no. 2, pp. 527-540, 1999.

[2] M. Brettel, N. Friederichsen, M. Keller, and M. Rosenberg, "How virtualization, decentralization and network building change the manufacturing landscape: An industry 4.0 perspective," International Journal of Mechanical, Industrial Science and Engineering, vol. 8, no. 1, pp. 3744, 2014.

[3] D. Lucke, C. Constantinescu, and E. Westkämper, "Smart factory-a step towards the next generation of manufacturing," Manufacturing systems and technologies for the new frontier, pp. 115-118, 2008.

[4] S. Wang, J. Wan, D. Li, and C. Zhang, "Implementing smart factory of industrie 4.0: an outlook," International Journal of Distributed Sensor Networks, vol. 12, no. 1, p. 3159805, 2016.

[5] E. Hofmann and M. Rüsch, "Industry 4.0 and the current status as well as future prospects on logistics," Computers in Industry, vol. 89, pp. $23-$ 34, 2017.

[6] J. Lee, B. Bagheri, and H.-A. Kao, "A cyber-physical systems architecture for industry 4.0-based manufacturing systems," Manufacturing Letters, vol. 3, pp. 18-23, 2015.

[7] F. Meng, D. Tan, and Y. Wang, "Development of agent for reconfigurable assembly system with jade," Proceedings of the 6th World Congress on Intelligent Control and Automation, pp. 7915-19, 2006.

[8] A. Koestler, "Beyond atomism and holism - the concept of the holon," Perspectives in Biology and Medicine, vol. 13, no. 2, pp. 131-154, 1970.

[9] H. Van Brussel, J. Wyns, P. Valckenaers, L. Bongaerts, and P. Peeters, "Reference architecture for holonic manufacturing systems: Prosa," Computers in Industry, vol. 37, pp. 255-274, 1998.

[10] P. Leitão and F. Restivo, "Adacor: A holonic architecture for agile and adaptive manufacturing control," Computers in Industry, vol. 57, no. 2, pp. 121-130, 2006.

[11] P. Valckenaers and H. Van Brussel, Design for the Unexpected From Holonic Manufacturing Systems towards a Humane Mechatronics Society. Butterworth-Heinemann, 1st edition ed., 2015.

[12] M. Kotzé, "Modular control of a reconfigurable conveyer system," Master's thesis, Stellenbosch University, South Africa, December 2016.

[13] I. F. Vis, "Survey of research in the design and control of automated guided vehicle systems," European Journal of Operational Research, vol. 170, no. 3, pp. 677-709, 2006.

[14] S. J. Guy, J. Chhugani, C. Kim, N. Satish, M. Lin, D. Manocha, and P. Dubey, "Clearpath: highly parallel collision avoidance for multi-agent simulation," in Proceedings of the 2009 ACM SIGGRAPH/Eurographics Symposium on Computer Animation, pp. 177-187, ACM, 2009.

[15] J. Van Den Berg, S. Guy, M. Lin, and D. Manocha, "Reciprocal n-body collision avoidance," Robotics Research, pp. 3-19, 2011.

[16] F. Tedesco, D. M. Raimondo, A. Casavola, and J. Lygeros, "Distributed collision avoidance for interacting vehicles: a command governor approach," IFAC Proceedings Volumes, vol. 43, no. 19, pp. 293-298, 2010.

[17] Y. Kuwata and J. P. How, "Cooperative distributed robust trajectory optimization using receding horizon MILP," IEEE Transactions on Control Systems Technology, vol. 19, no. 2, pp. 423-431, 2011.

[18] T. Mercy, R. Van Parys, and G. Pipeleers, "Spline-based motion planning for autonomous guided vehicles in a dynamic environment," IEEE Transactions on Control Systems Technology, vol. PP, no. 99, pp. 18, 2017.
[19] R. Van Parys and G. Pipeleers, "Distributed MPC for multi-vehicle systems moving in formation," Robotics and Autonomous Systems, vol. 97, pp. 144-152, 2017.

[20] R. Van Parys and G. Pipeleers, "Distributed model predictive formation control with inter-vehicle collision avoidance," in Proceedings of the 2017 Asian Control Conference, pp. 2399-2404, December 2017.

[21] R. Van Parys, M. Verbandt, and T. Mercy, "Ourbot." https://github.com/ meco-group/ourbot 2016.

[22] P. Beinschob and C. Reinke, "Graph slam based mapping for AGV localization in large-scale warehouses," in Intelligent Computer Соттиnication and Processing (ICCP), 2015 IEEE International Conference on, pp. 245-248, IEEE, 2015.

[23] J. Borenstein, H. R. Everett, L. Feng, and D. K. Wehe, "Mobile robot positioning: Sensors and techniques," 1997.

[24] D. Stojanović and N. Stojanović, "Indoor localization and tracking: Methods, technologies and research challenges," Facta Universitatis, Series: Automatic Control and Robotics, vol. 13, no. 1, pp. 57-72, 2014.

[25] A. Yassin, Y. Nasser, M. Awad, A. Al-Dubai, R. Liu, C. Yuen, R. Raulefs, and E. Aboutanios, "Recent advances in indoor localization: A survey on theoretical approaches and applications," IEEE Communications Surveys \& Tutorials, vol. 19, no. 2, pp. 1327-1346, 2016.

[26] M. Brezak, I. Petrović, and E. Ivanjko, "Robust and accurate global vision system for real time tracking of multiple mobile robots," Robotics and Autonomous Systems, vol. 56, no. 3, pp. 213-230, 2008.

[27] M. Velasco-Villa, E. Aranda-Bricaire, H. Rodríguez-Cortés, and J. González-Sierra, "Trajectory tracking for awheeled mobile robot using a vision based positioning system and an attitude observer," European Journal of Control, vol. 18, no. 4, pp. 348-355, 2012.

[28] W. L. Roque and D. Doering, "Trajectory planning for lab robots based on global vision and voronoi roadmaps," Robotica, vol. 23, no. 4 pp. 467-477, 2005.

[29] Y. Ji, A. Yamashita, and H. Asama, "Indoor positioning system based on distributed camera sensor networks for mobile robot," in International Conference on Intelligent Autonomous Systems, pp. 1089-1101, Springer, 2016.

[30] R. Van Parys and M. Verbandt, "Project Eagle." https://github.com/ meco-group/ProjectEagle 2017.

[31] B. E. Ilon, "Wheels for a course stable selfpropelling vehicle movable in any desired direction on the ground or some other base," April 1975. US Patent 3,876,255.

[32] P. Soetens, A Software Framework for Real-Time and Distributed Robot and Machine Control. PhD thesis, Department of Mechanical Engineering, KU Leuven, Belgium, May 2006.

[33] S. Boyd and L. Vandenberghe, Convex optimization. Cambridge university press, 2004.

[34] C. De Boor, "A practical guide to splines, revised edition, vol. 27 of applied mathematical sciences," 2001.

[35] R. Van Parys and T. Mercy, "OMG-tools." https://github.com/ meco-group/omg-tools 2016.

[36] J. Andersson, A general-purpose software framework for dynamic optimization. PhD thesis, Department of Electrical Engineering, KU Leuven, Belgium, 2013.

[37] Z. Zhang, "A flexible new technique for camera calibration," IEEE Transactions on pattern analysis and machine intelligence, vol. 22, no. 11 , pp. 1330-1334, 2000.

[38] O. Sorkine, "Least-squares rigid motion using svd," Technical notes, vol. 120 , no. 3, p. 52, 2009.

[39] J. Bruce and M. Veloso, "Fast and accurate vision-based pattern detection and identification," in Robotics and Automation, 2003. Proceedings. ICRA'03. IEEE International Conference on, vol. 1, pp. 1277-1282, IEEE, 2003.

[40] G. Klančar, M. Kristan, S. Kovačič, and O. Orqueda, "Robust and efficient vision system for group of cooperating mobile robots with application to soccer robots," ISA transactions, vol. 43, no. 3, pp. 329342,2004

[41] G. Bradski, "The opencv library," Dr. Dobb's Journal: Software Tools for the Professional Programmer, vol. 25, no. 11, pp. 120-123, 2000.

[42] P. Hintjens and L. Boccassi, "Zyre." https://github.com/zeromq/zyre 2017. 